\title{
Gap-mode Plasmonic Nanocavity
}

\section{Citation}

Russell, Kasey J., and Evelyn L. Hu. 2010. Gap-mode plasmonic nanocavity. Applied Physics Letters 97, no. 16: 163115.

\section{Published Version}

10.1063/1.3505154

\section{Permanent link}

http://nrs.harvard.edu/urn-3:HUL.InstRepos:11005282

\section{Terms of Use}

This article was downloaded from Harvard University's DASH repository, and is made available under the terms and conditions applicable to Other Posted Material, as set forth at http:// nrs.harvard.edu/urn-3:HUL.InstRepos:dash.current.terms-of-use\#LAA

\section{Share Your Story}

The Harvard community has made this article openly available.

Please share how this access benefits you. Submit a story.

Accessibility 


\title{
Gap-mode plasmonic nanocavity
}

\author{
Kasey J. Russell ${ }^{\mathrm{a})}$ and Evelyn L. Hu ${ }^{\mathrm{b})}$ \\ Harvard University School of Engineering and Applied Sciences, Harvard University, \\ 304 McKay Laboratory, Cambridge, Massachusetts 02138, USA
}

(Received 19 August 2010; accepted 1 October 2010; published online 21 October 2010)

\begin{abstract}
Here we describe the fabrication and characterization of a plasmonic nanocavity formed in the narrow gap between a $\mathrm{Ag}$ nanowire and a flat $\mathrm{Ag}$ substrate. The fluorescence spectrum of nanocrystals within the gap was strongly modified by the cavity modes, showing peaks of position and width $(\mathrm{Q} \sim 30-60)$ in quantitative agreement with numerical calculations. At gap spacings of $\sim 15 \mathrm{~nm}$, the noncavity background fluorescence is largely quenched by the Ag substrate, while the modal fluorescence remains strong, indicating that gap-type structures are more robust to fluorescence quenching. (C) 2010 American Institute of Physics. [doi:10.1063/1.3505154]
\end{abstract}

Optical cavities can tightly confine light in the vicinity of optical emitters, enhancing the interaction of light and matter. Such cavities have primarily been fabricated in dielectric materials to maximize the cavity quality and quality factors (Q) in excess of $10^{6}$ have been achieved in cavities with coupled emitters. ${ }^{1}$ It has been recently shown theoretically, ${ }^{2-5}$ however, that metal-based optical cavities utilizing surface plasmon polaritons (SPPs) (i.e., plasmonic cavities) can have sufficiently small mode volume (V) to achieve high $\mathrm{Q} / \mathrm{V}$ (the quantity relevant for high Purcell factors) despite their inherently lower Q. Here we describe such a nanocavity, formed in the gap between a Ag nanowire (NW) and a planar Ag substrate. The fluorescence spectrum of $\mathrm{PbS}$ nanocrystals (NCs) within the gap was strongly modified by the cavity modes, and bright emission from cavities with gaps as narrow as $15 \mathrm{~nm}$ indicates that fluorescence quenching near the surface of metals ${ }^{6}$ can be mitigated through modal engineering.

Substantial enhancements of fluorescence signal and Raman-scattering cross section have been obtained by coupling optical emitters to roughened metal surfaces or ensembles of colloidal metallic nanoparticles. ${ }^{7}$ There have been comparatively few experimental demonstrations of individual plasmonic cavities with coupled optical emitters, ${ }^{8-15}$ and of these, the only one to show substantial modification of the emitter fluorescence spectrum was a hybrid cavity formed from a dielectric NW and coupled planar metal layer. $^{8}$

The cavities reported here were formed by placing a Ag NW ( $\sim 70 \mathrm{~nm}$ diameter, $\sim 1-3 \mu \mathrm{m}$ in length) in close proximity to a $\mathrm{Ag}$ substrate $(\sim 15-70 \mathrm{~nm}$ separation), with the NW axis parallel to the substrate surface (Fig. 1). The spacing between NW and substrate was formed by one to two monolayers of PbS NCs (formed on a water subphase ${ }^{16}$ and stamped onto the sample ${ }^{17}$ ), clad by a layer of sputtered $\mathrm{SiN}$ on either side. The substrate is thus covered in an approximately uniform, encapsulated layer of NCs that, when not coupled to a cavity, have a nonpolarized fluorescence signal that is spectrally broad and featureless. Because the noncavity signal was nonpolarized, it was easily removed from the data, as will be discussed later. The excitation laser

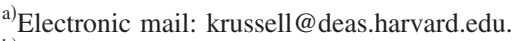

${ }^{b)}$ Electronic mail: ehu@seas.harvard.edu.
}

$(\lambda=633 \mathrm{~nm}$, typical power $1-20 \mu \mathrm{W})$, incident normal to the substrate, was focused onto the sample using a $100 \times, 0.5$ NA microscope objective. The luminescence signal was collected through the same objective, passed through a halfwave plate, a linear polarizer and a long-pass filter, and coupled into a grating spectrograph with liquid N2-cooled charge-coupled device camera.

Surface plasmon modes from the NW and substrate hybridize across the separation, forming a gap mode with
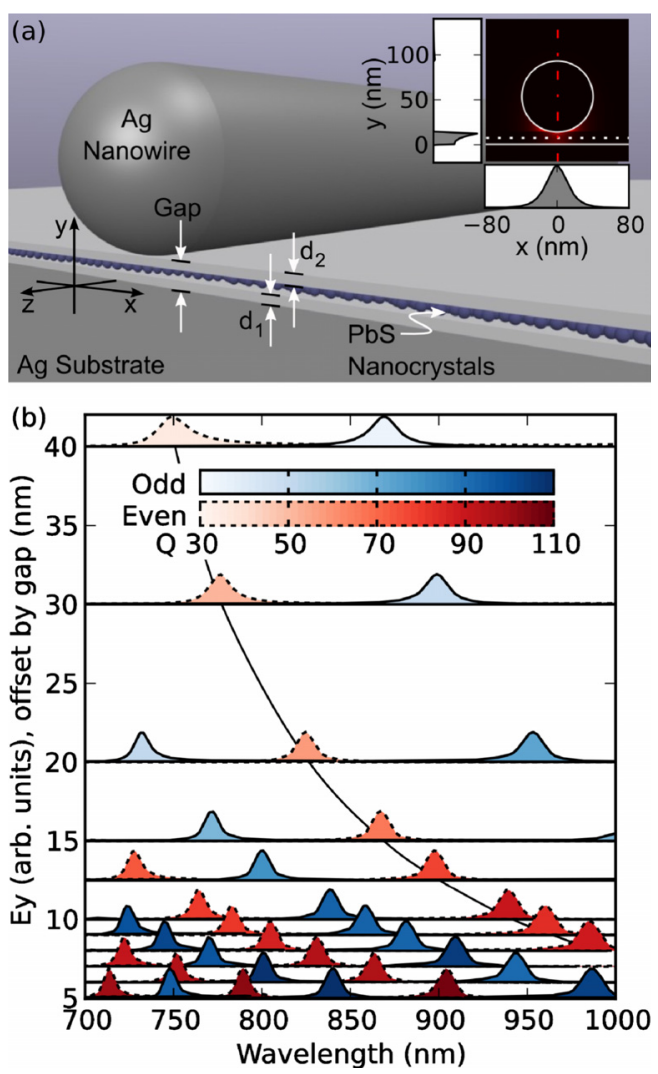

FIG. 1. (Color online) Nanowire-based gap-mode plasmonic nanocavity. (a) The cavity is comprised of a Ag NW and a Ag substrate, separated by a $\sim 5 \mathrm{~nm}$ thick layer of fluorescent $\mathrm{PbS}$ NCs and layers of insulating dielectric of thickness $d_{1}$ and $d_{2}$ (for total gap of $\sim 5 n m+d_{1}+d_{2}$ ). Inset shows calculated electric field intensity in the $x-y$ plane of a cavity with $14 \mathrm{~nm}$ gap. Cross-sectional field plots (along the broken lines) indicate that the field is tightly confined in the gap region, leading to strong overlap with the NCs. (b) Calculated resonances of $\sim 1 \mu \mathrm{m}$ long cavities of varying gap, labeled according to vector parity in the $\mathrm{z}$-direction. 
slower phase velocity [and thus larger effective refractive index $\left.\left(\mathrm{n}_{\text {eff }}\right)\right]$ than that of the isolated surface plasmon modes. ${ }^{6}$ Electromagnetic energy is confined within the gap in the y-direction (perpendicular to the substrate surface) by the opposing Ag surfaces. Confinement in the lateral ( $\mathrm{x}$ and $\mathrm{z}$ ) directions is provided by the index and mode mismatch that occurs approximately at the geometric boundaries of the NW. Because of the strong confinement in the $\mathrm{y}$ and $\mathrm{x}$ directions, only the lowest-order modes are observed in those directions while the cavity is sufficiently long in the z-direction to support multiple modes. Compared to plasmonic cavities formed on the surface of a single metal layer (such as a NW), these gap structures provide additional confinement in the direction normal to the metal surface, leading to better emittermode coupling ${ }^{6}$ and smaller effective mode volume $\mathrm{V}_{\text {eff }}$. Using finite-difference time-domain (FDTD) calculations of the samples with $15 \mathrm{~nm}$ gap, we determined the mode volume $\mathrm{V}$ to be of order $10^{-5} \mu \mathrm{m}^{3}$, which is comparable to what has been calculated for other plasmonic nanocavity designs. ${ }^{4}$ $\mathrm{V}_{\text {eff }} \sim 10^{-4}\left(\lambda / \mathrm{n}_{\text {eff }}\right)^{3}$, where $\lambda \sim 1 \mu \mathrm{m}$ is the vacuum wavelength and $n_{\text {eff }} \sim 2.3$ (as determined from the spacing between relative electric field maxima within the cavity). This yields $\mathrm{Q} / \mathrm{V}_{\text {eff }}$ of order $10^{5}$ for our cavities, which compares well with dielectric microcavities with $\mathrm{Q} \sim 5000$ and $\mathrm{V}_{\text {eff }}$ $\sim 5(\lambda / \mathrm{n})^{3}$ that have exhibited the Purcell effect. ${ }^{18}$

FDTD calculations of our cavities predicted that they should exhibit a series of Fabry-Perot resonances in the z-direction (parallel to the NW axis; see Fig. 1). As the gap between NW and substrate decreases, $\mathrm{n}_{\text {eff }}$ increases, shifting individual resonances to longer wavelengths $(\lambda)$. In Fig. 1(b), this evolution versus gap for a single even mode is illustrated using a line to guide the eye. Also indicated for each resonance in Fig. 1(b) is the respective Q (as determined by harmonic inversion ${ }^{19,20}$ of the time-dependent electric field). As the gap decreases, Q continually increases, even though the fraction of electromagnetic energy contained within the metal is increasing. This suggests that $\mathrm{Q}$ is limited not by losses within the metal but by reflection losses at the ends of the cavity, which decrease as $\mathrm{n}_{\text {eff }}$ (and thus the index mismatch at the cavity boundary) increases. Decreasing V results in a simultaneous increase in $\mathrm{Q}$. This also suggests that it should be possible to increase $Q$ further by engineering a cavity with lower reflection losses-without sacrificing small mode volume.

Coupling to modes of the cavities dramatically altered the fluorescence spectrum of the PbS NCs, as can be seen in Fig. 2(a). A series of prominent peaks appeared, with both the wavelength and Q of the resonances in good agreement with FDTD calculations of the corresponding cavity modes. (Typical Q was in the range 40-60). In addition, the luminescence associated with these peaks was highly linearly polarized parallel to the NW axis [see Fig. 2(e)], in agreement with FDTD calculations of the far-field radiation from a cavity (not shown). Because the cavity fluorescence was polarized while the noncavity fluorescence was not, we were able to isolate each cavity spectrum by subtracting the luminescence polarized perpendicular to the NW axis from that polarized parallel. Also illustrated in Fig. 2(e) is an issue that complicated our measurements as follows: a general, permanent, decrease in cavity fluorescence over time. The data presented in Fig. 2(e) was collected by rotating the polarizer in the direction indicated, and it can be seen that the intensity
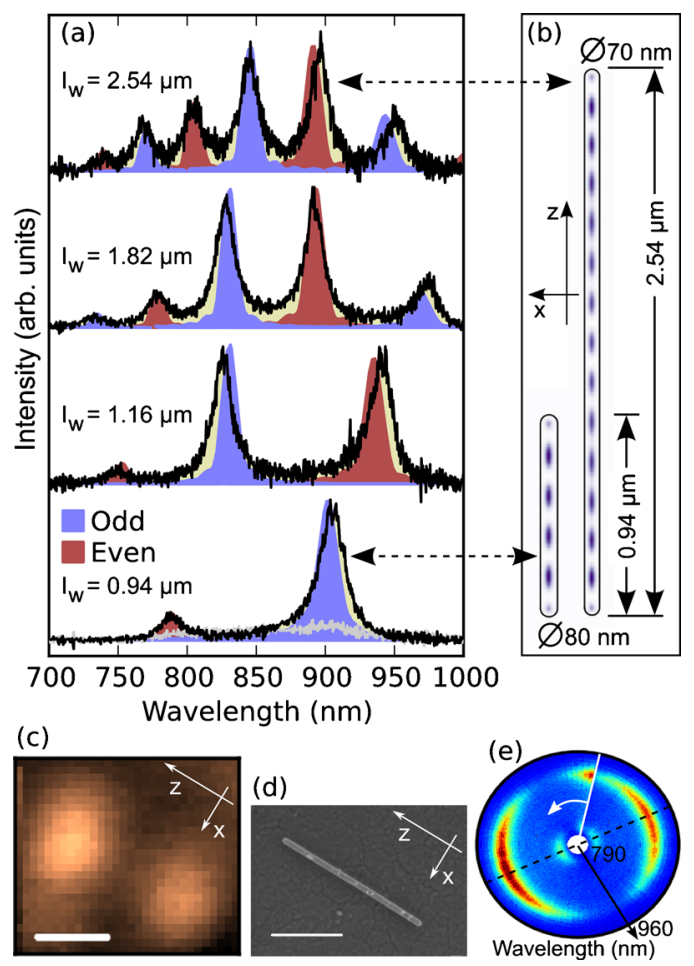

FIG. 2. (Color online) Plasmonic nanocavity with $15 \mathrm{~nm}$ gap. (a) Measured fluorescence spectra (lines) and FDTD calculated resonances (filled curves) from four cavities of different lengths from sample A. Each spectrum is labeled with the corresponding NW length $\left(1_{\mathrm{w}}\right)$. Measured cavity spectra were corrected for noncavity background fluorescence (e.g., gray line, $0.94 \mu \mathrm{m}$ long cavity). The FDTD simulations differed only in NW length and diameter, agreeing with measured NW length within $2 \%$ and with radius within the measurement error. (b) Calculated electric field intensity profile in the center of the gap for the indicated modes. (c) Excitation map and (d) SEM micrograph of the $1.82 \mu \mathrm{m}$ long cavity from (a). Scale bar is $1 \mu \mathrm{m}$. (e) Fluorescence spectrum vs polarization angle from a $0.93 \mu \mathrm{m}$ long cavity. Dashed line indicates NW orientation, white line and arrow indicate the start and direction of the sweep.

of the bright mode had decreased substantially over the course of the $\sim 11$ min needed to acquire all of the spectra. We have not yet determined the cause of this decrease.

The NCs within a cavity are most likely excited through a combination of direct laser illumination and secondary excitation by neighboring, uncoupled, and photoexcited NCs. The neighboring NCs (which are also responsible for the noncavity fluorescence) may lead to excitation of cavity NCs either through fluorescence or through generation of SPPs that propagate along the surface of the $\mathrm{Ag}$ substrate and are absorbed by the cavity $\mathrm{NCs}{ }^{21}$ All of these mechanisms are likely to be most efficient when the laser spot is positioned over the end of a cavity, since being incident from the end of the cavity provides for better wave vector and mode matching with the cavity modes. This was observed experimentally, as shown in Figs. 2(c) and 2(d).

An interesting feature of our cavities is the improved cavity emission for smaller gap separations. Calculations by Ford and Weber ${ }^{6}$ on single Ag structures predict fluorescence quenching for emitters placed less than $\sim 10 \mathrm{~nm}$ from the metal. As has been previously observed, ${ }^{9}$ an emitter placed very close to a flat metal region can generate both SPPs and "lossy surface waves" (LSW), surface excitations whose long wave vector prevents them from being excited via farfield radiation. Of these, the SPP can have long propagation constants and can be coupled, for example, via scattering, ${ }^{9}$ 

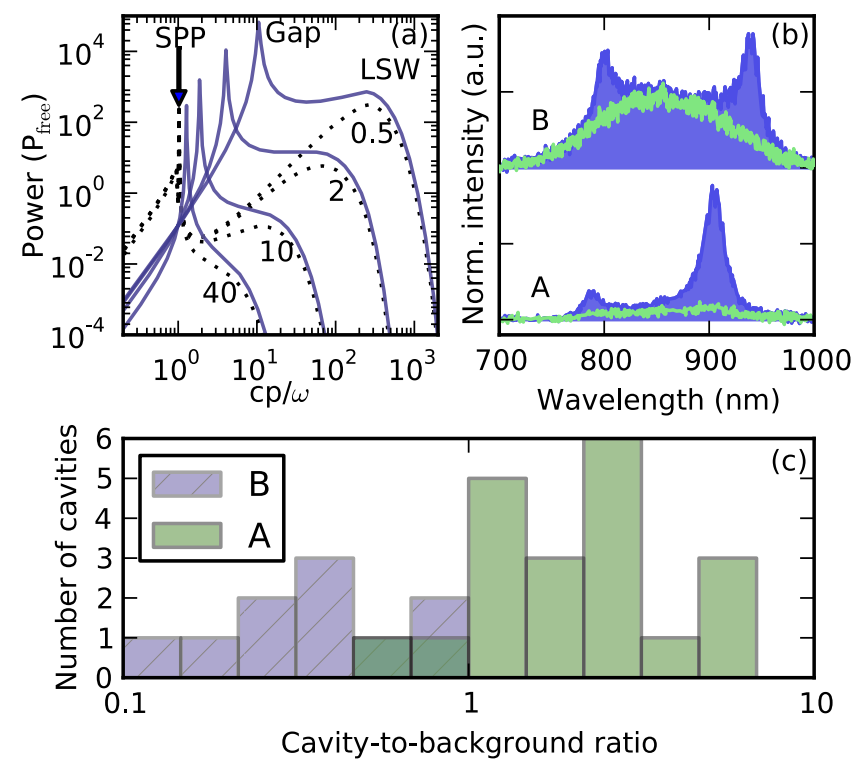

FIG. 3. (Color online) Resistance of gap mode to fluorescence quenching. (a) Calculations of emitted power at $\hbar \omega=1.96 \mathrm{eV}$ for a perpendicular dipole near a flat Ag surface (dotted) or between two parallel Ag surfaces (solid) as a function of the wave vector component parallel to the surface, p. Dipolemetal distance is indicated in nanometer; calculations are normalized to free-space dipole power spectrum, $\mathrm{P}_{\text {free. }}$ (b) Example fluorescence spectra from cavities with different NC-substrate spacings (total gap thickness of sample A, $\sim 15 \mathrm{~nm} ; \mathrm{B}, \sim 40 \mathrm{~nm})$ showing emission polarized parallel (filled curve) and perpendicular (line) to the NW axis. (c) Ratio of integrated cavity emission to integrated background emission for all devices measured from the two samples. [For the example spectra plotted in (b), this ratio is visually given by the area of the part of the filled curve above the green line divided by the area of the part below.] Note that the horizontal scale is logarithmic; values above one indicate that the cavity fluorescence is brighter than the background.

into far-field radiation. LSW, in contrast, are believed to be almost entirely absorbed by the metal and lost as heat. ${ }^{6}$ In the case of a single Ag region, the power emitted into the LSW increases greatly as the emitter is placed closer to the metal while that emitted into the surface plasmon resonance remains relatively unchanged [Fig. 3(a)].

We compared two samples, A and B, that differed only in the thicknesses of the lower dielectric layer $\left[A, d_{1}=5 \mathrm{~nm} ; B\right.$, $\mathrm{d}_{1}=30 \mathrm{~nm}$; Figs. 3(b) and 3(c)]. The NCs of sample A are close enough to the Ag substrate that their fluorescence is partially quenched through the excitation of SPP and LSW. ${ }^{6}$ In sample B, in contrast, the NCs are far enough from the substrate to largely avoid quenching. In agreement with these reports, we found the noncavity fluorescence of sample A to be lower than that of sample B. However, if we analyze the relative intensity of the cavity modes with respect to the noncavity background fluorescence, we find that the background fluorescence from sample B is brighter than the cavity modes [Figs. 3(b) and 3(c)]. For sample A, with the smaller gap separation, the opposite is true; the cavity mode fluorescence is often several times brighter than the background. The cavities we measured were oriented randomly, and we saw no performance difference for different cavity orientations relative to the (fixed) laser polarization. This suggests that the greatly enhanced luminescence we observe at each of the cavity modes is not simply the result of enhanced in-coupling of the laser excitation, since the cavity modes are known to be strongly polarized. In addition, nu- merical calculations of emission angle (not shown) reveal only a weak dependence on cavity gap thickness. This indicates that the collection efficiency of our microscope objective is not significantly different for emission from the cavity modes of samples A and B.

Instead, we can understand the difference between samples A and B as illustrating the power of gap-mode structures; Ford and Weber showed that emission into the gap mode between two closely-spaced metal regions increases greatly as the gap shrinks. We have extended their analysis, calculating the power spectrum of the gap structure in the nonlocal approximation to investigate the relative generation of LSW and gap-mode plasmon polaritons [Fig. 3(a)]. Qualitatively, it appears that as the gap shrinks, emission into the gap mode increases at nearly the same rate as emission into the LSW. This implies that emission into the gap mode should be resistant to quenching at much closer emitter-metal spacings, resulting in greatly enhanced emitter-mode overlap and greatly decreased mode volume. This has been borne out by our measurements. [A more quantitative analysis of the emission characteristics will require integration of the power spectra of Fig. 3(a) while distinguishing between LSW and gap-mode excitations at the same wave vector. We are currently investigating this.] The robustness to quenching of these gap structures has important implications for further miniaturization of plasmonic nanocavities.

The authors acknowledge support from NSF/NSEC (NSF/PHY-06-46094), the use of NSF/NNIN facilities at Harvard University's Center for Nanoscale Systems (CNS), the use of the hpc computer cluster at Harvard, and fabrication assistance from John Joo.

${ }^{1}$ P. Bianucci, X. Wang, J. G. Veinot, and A. Meldrum, Opt. Express 18, 8466 (2010).

${ }^{2}$ S. Maier, Opt. Express 14, 1957 (2006).

${ }^{3}$ Y. C. Jun, R. D. Kekatpure, J. S. White, and M. L. Brongersma, Phys. Rev. B 78, 153111 (2008).

${ }^{4}$ M. Seo, S. Kwon, H. Ee, and H. Park, Nano Lett. 9, 4078 (2009).

${ }^{5}$ Y. Gong and J. Vučković, Appl. Phys. Lett. 90, 033113 (2007).

${ }^{6}$ G. Ford and W. Weber, Phys. Rep. 113, 195 (1984).

${ }^{7}$ M. Moskovits, Rev. Mod. Phys. 57, 783 (1985).

${ }^{8}$ R. F. Oulton, V. J. Sorger, T. Zentgraf, R. Ma, C. Gladden, L. Dai, G. Bartal, and X. Zhang, Nature (London) 461, 629 (2009).

${ }^{9}$ Y. Fedutik, V. V. Temnov, O. Schops, U. Woggon, and M. V. Artemyev, Phys. Rev. Lett. 99, 136802 (2007).

${ }^{10}$ D. K. Gifford and D. G. Hall, Appl. Phys. Lett. 81, 4315 (2002).

${ }^{11}$ A. V. Akimov, A. Mukherjee, C. L. Yu, D. E. Chang, A. S. Zibrov, P. R. Hemmer, H. Park, and M. D. Lukin, Nature (London) 450, 402 (2007).

${ }^{12}$ Y. Gong, J. Lu, S. Cheng, Y. Nishi, and J. Vučković, Appl. Phys. Lett. 94, 013106 (2009).

${ }^{13}$ Y. C. Jun, R. Pala, and M. L. Brongersma, J. Phys. Chem. C 114, 7269 (2010).

${ }^{14}$ Y. Gong, S. Yerci, R. Li, L. Dal Negro, and J. Vučković, Opt. Express 17, 20642 (2009).

${ }^{15}$ E. J. A. Kroekenstoel, E. Verhagen, R. J. Walters, L. Kuipers, and A. Polman, Appl. Phys. Lett. 95, 263106 (2009).

${ }^{16}$ V. Santhanam, J. Liu, R. Agarwal, and R. P. Andres, Langmuir 19, 7881 (2003).

${ }^{17}$ J. L. Wilbur, A. Kumar, E. Kim, and G. M. Whitesides, Adv. Mater. (Weinheim, Ger.) 6, 600 (1994).

${ }^{18}$ J. M. Gérard, B. Sermage, B. Gayral, B. Legrand, E. Costard, and V. Thierry-Mieg, Phys. Rev. Lett. 81, 1110 (1998).

${ }^{19}$ V. A. Mandelshtam and H. S. Taylor, J. Chem. Phys. 107, 6756 (1997).

${ }^{20} \mathrm{~S}$. Johnson, HARMINV, http://ab-initio.mit.edu/wiki/index.php/Harminv.

${ }^{21}$ A. L. Falk, F. H. L. Koppens, C. L. Yu, K. Kang, N. de Leon Snapp, A. V. Akimov, M. Jo, M. D. Lukin, and H. Park, Nat. Phys. 5, 475 (2009). 